\title{
The microbiota continuum along the female reproductive tract and its relation to uterine-related diseases
}

\author{
Chen Chen ${ }^{1,2}$, Xiaolei Song ${ }^{1,3}$, Weixia Wei ${ }^{4,5}$, Huanzi Zhong (10) 1,2,6, Juanjuan Dai ${ }^{4,5}$, Zhou Lan', Fei Li ${ }^{1,2,3}$, \\ Xinlei $\mathrm{Yu}^{1,2}$, Qiang Feng ${ }^{1,7}$, Zirong Wang ${ }^{1}$, Hailiang Xie ${ }^{1}$, Xiaomin Chen ${ }^{1}$, Chunwei Zeng ${ }^{1}$, Bo Wen ${ }^{1,2}$, \\ Liping Zeng ${ }^{4,5}$, Hui Du ${ }^{4,5}$, Huiru Tang ${ }^{4,5}$, Changlu Xu1,8, Yan Xia1,3, Huihua Xia ${ }^{1,2,9}$, Huanming Yang ${ }^{1,10}$, \\ Jian Wang ${ }^{1,10}$, Jun Wang ${ }^{1,11}$, Lise Madsen (1) ${ }^{1,6,12}$, Susanne Brix (10) ${ }^{13}$, Karsten Kristiansen ${ }^{1,6}$, Xun Xu ${ }^{1,2}$, \\ Junhua Li (iD) 1,2,9,14, Ruifang Wu ${ }^{4,5}$ \& Huijue Jia (i) ${ }^{1,2,9,11}$
}

Reports on bacteria detected in maternal fluids during pregnancy are typically associated with adverse consequences, and whether the female reproductive tract harbours distinct microbial communities beyond the vagina has been a matter of debate. Here we systematically sample the microbiota within the female reproductive tract in 110 women of reproductive age, and examine the nature of colonisation by $16 \mathrm{~S}$ rRNA gene amplicon sequencing and cultivation. We find distinct microbial communities in cervical canal, uterus, fallopian tubes and peritoneal fluid, differing from that of the vagina. The results reflect a microbiota continuum along the female reproductive tract, indicative of a non-sterile environment. We also identify microbial taxa and potential functions that correlate with the menstrual cycle or are overrepresented in subjects with adenomyosis or infertility due to endometriosis. The study provides insight into the nature of the vagino-uterine microbiome, and suggests that surveying the vaginal or cervical microbiota might be useful for detection of common diseases in the upper reproductive tract.

\footnotetext{
${ }^{1}$ BGI-Shenzhen, Shenzhen 518083, China. ${ }^{2}$ China National Genebank, BGI-Shenzhen, Shenzhen 518120, China. ${ }^{3}$ BGI Education Center, University of Chinese Academy of Sciences, Shenzhen 518083, China. ${ }^{4}$ Peking University Shenzhen Hospital, Shenzhen 518036, China. ${ }^{5}$ Shenzhen Key Laboratory on Technology for Early Diagnosis of Major Gynecological diseases, Shenzhen 518036, China. ${ }^{6}$ Department of Biology, Laboratory of Genomics and Molecular Biomedicine, University of Copenhagen, Universitetsparken 13, 2100 Copenhagen, Denmark. ${ }^{7}$ Shenzhen Engineering Laboratory of Detection and Intervention of human intestinal microbiome, BGI-Shenzhen, Shenzhen 518083, China. ${ }^{8}$ Qingdao University-BGI Joint Innovation College, Qingdao University, Qingdao 266071 , China. ${ }^{9}$ Shenzhen Key Laboratory of Human Commensal Microorganisms and Health Research, BGI-Shenzhen, Shenzhen 518083, China. ${ }^{10}$ James D. Watson Institute of Genome Sciences, Hangzhou 310000, China. ${ }^{11}$ Macau University of Science and Technology, Taipa, Macau 999078 , China. ${ }^{12}$ National Institute of Nutrition and Seafood Research, (NIFES), Postboks 2029, Nordnes, N-5817 Bergen, Norway. ${ }^{13}$ Department of Biotechnology and Biomedicine, Technical University of Denmark, Soltofts Plads, Building 221, 2800 Kongens Lyngby, Denmark. ${ }^{14}$ School of Bioscience and Biotechnology, South China University of Technology, Guangzhou 510006, China. Chen Chen, Xiaolei Song, Weixia Wei, Huanzi Zhong, Juanjuan Dai and Zhou Lan contributed equally to this work. Correspondence and requests for materials should be addressed to J.L. (email: lijunhua@genomics.cn) or to R.W. (email: wurf100@126.com) or to H.J. (email: jiahuijue@genomics.cn)
} 
$\mathrm{n}$ marsupials and placental mammals, the female reproductive tract has developed unique structures such as the vagina and the uterus. During the reproductive cycle, mature oocytes from the ovaries enter the peritoneal cavity to be captured by fimbriae of the fallopian tubes. The oocytes are fertilised in the fallopian tubes, and the zygotes develop and translocate to the uterus for implantation. While the vagina is home for trillions of bacteria, the uterus and the fallopian tubes are generally believed to be sterile, which would require the cervix to be a perfect barrier. Mucins in the cervix, however, are known to change conformation, leading to aggregations dependent on $\mathrm{pH}$ variations during the menstrual cycle ${ }^{1}$. Such changes may in theory allow passage of bacteria during certain conditions.

Lactobacilli are known as the keystone species of the vaginal microbiota in reproductive-age women ${ }^{2}$. Indeed, cultureindependent $16 \mathrm{~S}$ rRNA gene amplicon sequencing studies from the United States have identified 5 community types of vaginal microbiota, 3 or 4 of which contain $>90 \%$ Lactobacillus $^{3-5}$. The lactic acid produced by the vaginal microbiota helps maintain a low $\mathrm{pH}$ of 3.5-4.5, a major factor in limiting the growth of potentially harmful bacteria. Alterations in the vaginal microbiota play a role in common conditions such as bacterial vaginosis, sexually transmitted diseases, urinary infections and preterm birth $^{2}, 6,7$.

In contrast, the upper reproductive tract remains largely unexplored. Bacteria have mostly been studied in small sample sizes in the context of infection, especially in relation to preterm birth ${ }^{6}$. Although controversial, the placenta has recently been reported to harbour a microbiota ${ }^{8}$. It is as yet not clear what type of bacteria, if any, may exist in the upper reproductive tract of the vast majority of women who are not in periods of infection or pregnancy. Furthermore, it is not known if an upper reproductive tract microbiota could play a role in uterinerelated diseases such as hysteromyoma, adenomyosis and endometriosis.

Here, we systematically sampled the microbiota at six sites within the female reproductive tract, from a large cohort of Chinese women of reproductive age. Bacteria were identified by using 16S rRNA gene amplicon sequencing, real-time qPCR, as well as conventional bacterial culturing. The results indicate a continuity of the vagino-uterine microbiome, with a distinct trend within the same individual. Potential bacterial markers for adenomyosis and endometriosis were also identified.

\section{Results}

Microbiota composition at six sites within the female reproductive tract. To explore the microbiota beyond the vagina, we collected samples from six locations (CL, lower third of vagina; $\mathrm{CU}$, posterior fornix; $\mathrm{CV}$, cervical mucus drawn from the cervical canal; ET, endometrium; FLL and FRL, left and right fallopian tubes; PF, peritoneal fluid from the pouch of Douglas) throughout the female reproductive tract from an initial study cohort of 95 Chinese women submitted to surgery for conditions not known to involve infections (Fig. 1). These conditions included hysteromyoma (also known as uterine fibroids), adenomyosis, endometriosis and salpingemphraxis, which are to our knowledge the best proxies for the upper reproductive tract in healthy young women (Supplementary Data 1). Samples from the vagina and the cervical mucus (CL, CU, CV) were taken upon visit to the clinic (without any prior disturbance). Samples from the peritoneal and uterine sites (PF, FL, ET) were taken during laparoscopy or laparotomy from minimally invasive surgery cuts to avoid possible contamination from the vaginal microbiota if the samples were to go through the cervical os (Fig. 1, Supplementary Fig. 1). The samples were subjected to $16 \mathrm{~S}$ rRNA gene amplicon sequencing, quality-controlled and clustered into operational taxonomic units (OTUs) (Supplementary Data 2, 3).

The lower (CL, CU, CV) and upper (ET, FL, PF) reproductive tract samples separated when subjected to principal coordinate analyses (PCoA) based on weighted and unweighted UniFrac distances (Fig. 1a, b). The FLL and FRL showed essentially the same microbiota (Fig. 1a, b), and were therefore clustered into a common FL category for further analysis (except for calculation of intra-individual and inter-individual differences, referred to later).

Consistent with previous reports ${ }^{3-5}$, the $\mathrm{CL}$ as well as the $\mathrm{CU}$ were dominated by the Lactobacillus genus (Fig. 1c), and exhibited a low $\alpha$-diversity (Supplementary Fig. 2a). At the species level, the CL and CU samples contained L. crispatus, L. iners and other Lactobacillus spp. (Supplementary Fig. 2b), similar to previous reports from the $\mathrm{US}^{3-5}$. Notably, CV samples generally contained a lower proportion of Lactobacillus than the vaginal samples (Fig. 1c), and varied among the sampled individuals (Supplementary Fig. 3a). In the ET samples, Lactobacillus no longer dominated, and bacteria such as Pseudomonas, Acinetobacter, Vagococcus and Sphingobium constituted a notable fraction of the microbiota (Fig. 1c, Supplementary Fig. 3b). The proportion of these bacteria further increased at the openings of the FL, leaving a median relative abundance of $1.69 \%$ for Lactobacillus (Fig. 1c, Supplementary Fig. 3d). The PF samples generally lacked the presence of Lactobacillus, but otherwise harboured a microbiota as diverse but not completely the same as the FL samples (Fig. 1, Supplementary Fig. 3c).

At the phylum level, the Firmicutes-dominated lower reproductive tract microbiota contrasted the large proportions of Proteobacteria, Actinobacteria and Bacteroidetes in the upper reproductive tract (Supplementary Fig. 3e).

As noted above, we took samples from the uterine and peritoneal sites without going through the cervical os to address a major concern in the field that transcervical samples might be contaminated by the vaginal microbiota. We nonetheless tested the specificity of sampling route by collection of ET samples via the uterus as well as through the cervical os, and of CV from the cervical os as well as from the uterine end. The distribution of bacteria in the samples taken through the cervical os showed high similarity to that in samples taken by opening the uterus during surgery (Supplementary Fig. 4a, b), suggesting that both the uterine and cervical microbiota could be readily accessed and analysed in women not undergoing surgery.

Estimation of bacterial biomass in the female reproductive tract. In order to provide some absolute measure of the microbiota beyond the vagina, we developed a species-specific real-time qPCR approach, focusing on the dominant vaginal bacteria Lactobacilli. The abundances of L. crispatus, L. iners, L. gasseri and L. jensenii monotonously decreased from CL, CU, CV, to ET and PF (Supplementary Fig. 5a-d), consistent with genus level data from 16S rRNA gene amplicon sequencing (Fig. 1). The concordance between $\mathrm{qPCR}$ and amplicon sequencing results were further confirmed by Spearman's correlation, which showed a correlation coefficient of 0.72 for $L$. crispatus and 0.56 for L. iners, the two dominant Lactobacilli species (Supplementary Fig. 2b).

The total bacterial biomass within each site was then calculated based on the copy number from qPCR, divided by the corresponding relative abundance in the sample according to $16 \mathrm{~S}$ rRNA gene sequencing. This calculation revealed that, while the vaginal sites contained about four orders of magnitude more 
a

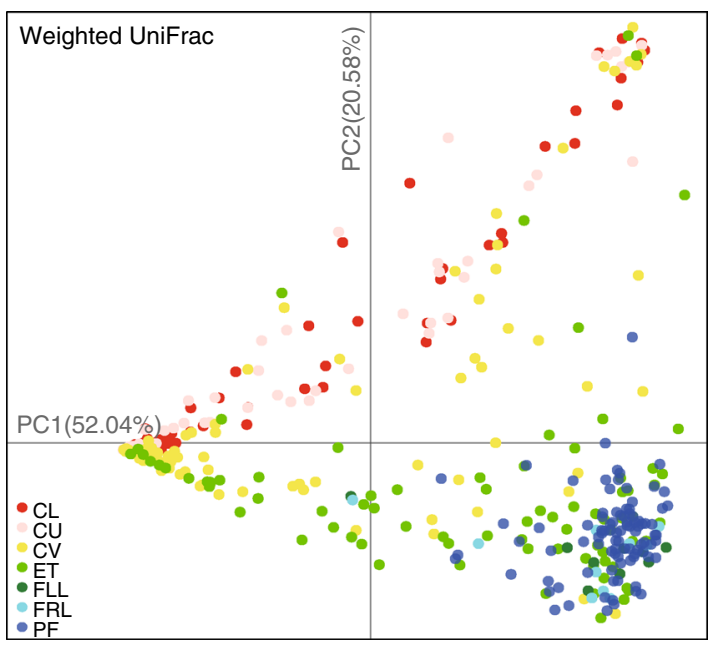

\section{b}

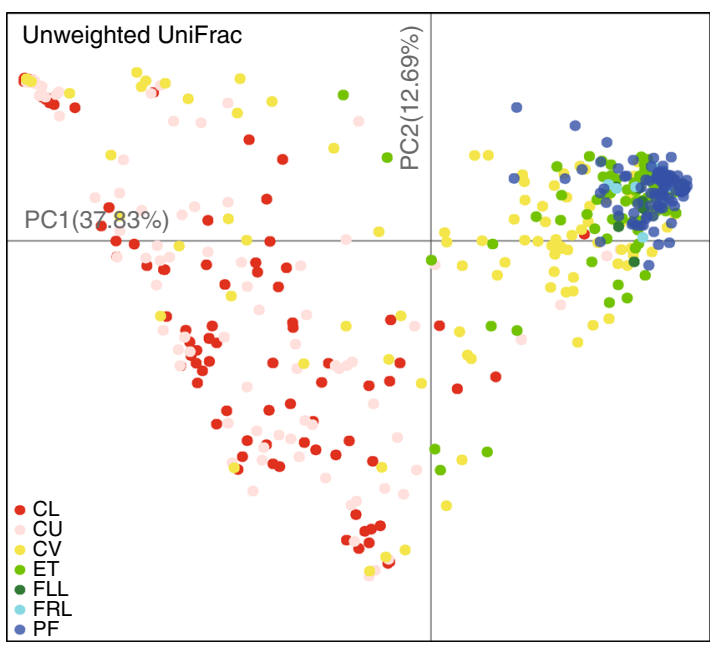

C

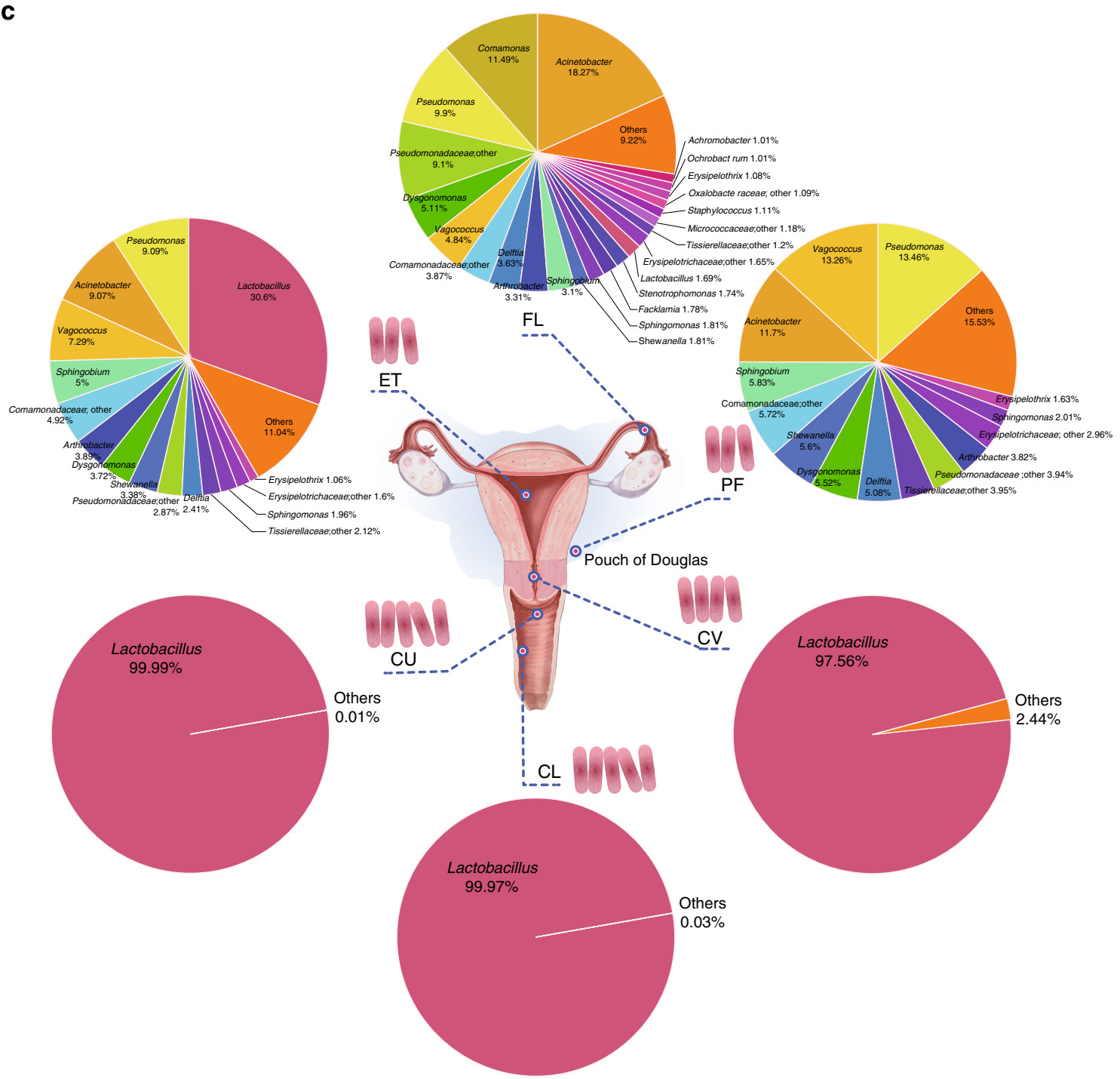

Fig. 1 Composition of the vagino-uterine microbiota. a, b PCoA on the samples based on weighted (a) and unweighted (b) UniFrac distances. Samples were taken from CL, CU and CV before operation, and from ET, FLL, FRL and PF during operation. Each dot represents one sample. $\mathbf{c}$ Pie chart for the microbial genera at each body site according to the median relative abundance. Genera that took up $<1 \%$ of the microbiota were labelled together as 'others'. A pink rod represents about $10^{2}$ copies/sample, according to the qPCR results in Supplementary Fig. 5e. Samples derive from the study cohort of 95 reproductive-age women ( $n=94 \mathrm{CL}, 95 \mathrm{CU}, 95 \mathrm{CV}, 80 \mathrm{ET}, 93 \mathrm{PF}, 9 \mathrm{FLL}$ and 10 FRL, Supplementary Data 1) 


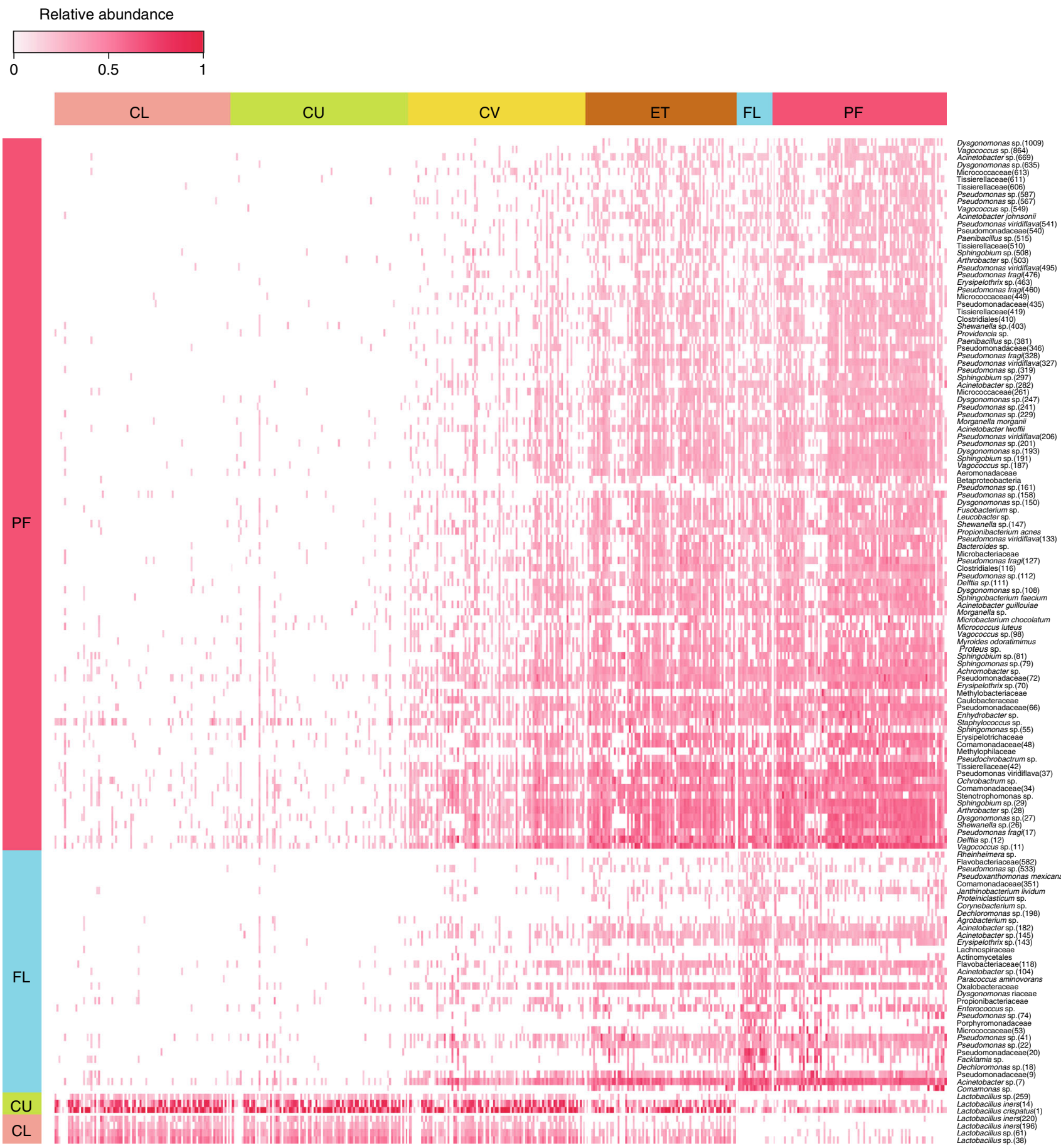

Fig. 2 Signature species for each body site. Heatmap for the relative abundances of signature OTUs from each body site $(P<0.05$, multi-level pattern analysis, and IndVal >0.5). Each bar represents one sample $(n=94 \mathrm{CL}, 95 \mathrm{CU}, 95 \mathrm{CV}, 80 \mathrm{ET}, 93 \mathrm{PF}, 9 \mathrm{FLL}$ and $10 \mathrm{FRL}$, Supplementary Data 1)

bacteria $\left(10^{10}-10^{11}\right)$, the PF samples contained similar bacterial biomass as the ET samples (Supplementary Fig. 5e), which are orders of magnitude above potential background noise ${ }^{9}$. The negative controls (sterile phosphate-buffered saline (PBS), sterile saline and ultrapure water) showed much higher cycle threshold (Ct values) than the low biomass ET and PF samples (Supplementary Table 1, Supplementary Fig. 1), signifying that much less bacterial DNA was detected in non-bio control samples. It is thus important to bear in mind that the higher bacterial diversity in the upper reproductive tract corresponds to a lower bacterial biomass than the much better established vaginal microbiota (Fig. 1c).
Cultivation of live bacteria from the upper reproductive tract. Another important question is whether live bacteria rather than debris makes up the bacterial DNA signal in the upper reproductive tract samples. Hence, in a validation study we performed 16S rRNA gene amplicon sequencing along with culturing of live bacteria from an additional cohort of 15 women.

Of the 21 diluent negative controls, 19 had lower DNA concentrations and library concentrations (close to zero) than their respective tissue samples (Supplementary Data 4), again confirming that there was little DNA in the non-bio control samples. In PCoA, microbial profiles differed between the low biomass upper reproductive tract samples and diluent controls 
a

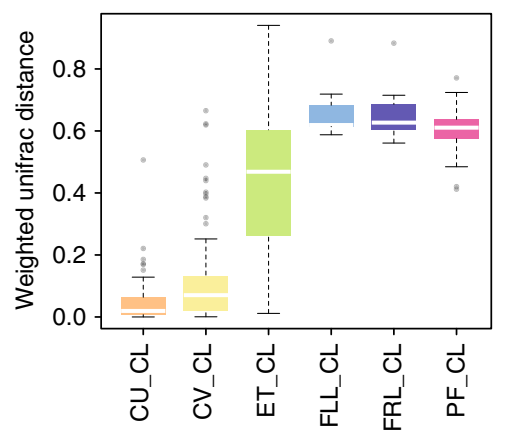

b

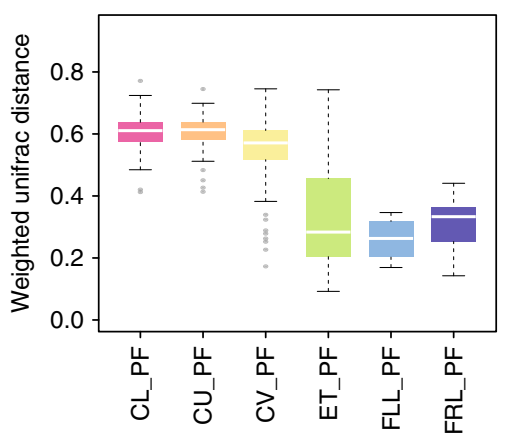

C

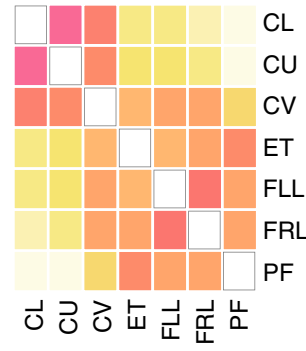

d

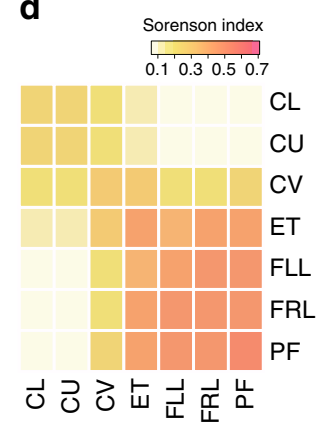

Fig. 3 Similarity of the vagino-uterine microbiota within and between individuals. a, b Weighted UniFrac distance of the microbiota at each body site relative to the $\mathrm{CL}(\mathbf{a})$ and $\mathrm{PF}(\mathbf{b})$ in the same individual. Boxes denote the interquartile range (IQR) between the first and third quartiles (25th and 75th percentiles, respectively), and the line inside the boxes denote the median. The whiskers denote the lowest and highest values within 1.5 times the IQR from the first and third quartiles, respectively. Circles represent data points beyond the whiskers. $\mathbf{c}$ Intra-individual Sorenson indices between different body sites. d Inter-individual Sorenson indices between different body sites. Samples derive from the study cohort of 95 reproductive-age women ( $n=94 \mathrm{CL}$, 95 CU, 95 CV , 80 ET, 93 PF, 9 FLL and 10 FRL, Supplementary Data 1)

(Supplementary Fig. 6, sterile saline for the peritoneal samples and PBS for the remaining samples).

The same samples extracted at different times, diluted or undiluted before amplification, or sequenced in different runs showed high reproducibility, indicating that random contamination had no major impact on bacterial community structure detected in the upper reproductive tract (Supplementary Table 2, Supplementary Data 5).

We further attempted to culture and isolate live bacteria from the freshly collected samples. PF samples were plated on peptone yeast extract-glucose (PYG) agar with 5\% horse blood and incubated aerobically or anaerobically. Positive bacterial cultures were obtained from 5 out of 15 subjects, resulting in 8 different isolates belonging to 7 genera such as Lactobacillus, Staphylococcus and Actinomyces (Supplementary Table 3). These genera were also detected in our $16 \mathrm{~S}$ rRNA gene amplicon sequencing data, and supported by previous cultivation of vaginal or amniotic samples ${ }^{10-16}$ (Supplementary Table 3). Reassuringly, no bacteria were isolated from the diluent-negative controls (including swabs from sterilised skin samples from the patients and the gloves used by doctors). Thus, live bacteria do exist in the upper reproductive tract, of which we succeeded in isolation of some using conventional culturing methods.

Signature species for each site of the reproductive tract. To get more insight into the ecology of the vagino-uterine communities, we defined signature OTUs for each body site considering both the occurrence and abundance of the given OTUs $(P<0.05$ in multi-level pattern analysis, and indicator value index (IndVal) $>0.5$, Fig. 2, Supplementary Data 6). L. iners and L. crispatus were identified as signature species of the vaginal sites (CL and CU). No signature OTUs with IndVal $>0.5$ was identified for $\mathrm{CV}$ or ET, suggesting that they are bacterial transition zones between the vagina and the upper anatomical sites. The FL had a number of signature OTUs including Pseudomonas, Erysipelothrix and Facklamia (Fig. 2, Supplementary Data 6). The PF featured many signature OTUs, including Pseudomonas, Morganella, Sphingobium and Vagococcus, all of which increased monotonously from the $\mathrm{CU}$ and the $\mathrm{CV}$ to the ET and PF (Supplementary Data 6). In support of the existence of these taxa in the upper reproductive tract, they have been reported in previous studies of the vaginal microbiota in humans or animals ${ }^{17-21}$. Overall, we observed the female reproductive tract as a habitat for facultative anaerobes or aerobes; the upper sites contained a variety of bacteria that grow in mildly alkaline conditions, contrasting the Lactobacillus-dominated low $\mathrm{pH}$ environment of the vagina.

Intra-individual and inter-individual similarity in the vaginouterine microbiota. To examine the microbiota relationship at the six sites of the reproductive tract, we computed the intra-individual and inter-individual distances between samples from each individual. Weighted intra-individual UniFrac distance relative to CL samples gradually increased from $\mathrm{CU}$ and $\mathrm{CV}$ to ET and PF (Fig. 3a), consistent with the anatomical contingency of these sites (Fig. 1). The same was also true in reverse order, i.e. weighted intra-individual UniFrac distance relative to PF gradually increased from ET, CV, to CU and CL (Fig. 3b). Samples from the same individual were highly correlated, and the Sorenson indices between body sites were consistent with their anatomical contingency (Fig. 3c). The intra-individual correlation was notable even between the less invasive sampling site of CV and the less reachable PF (median of Sorenson index $=0.255$, Fig. 3c), hinting at the possibility of minimally invasive surveys of the uterine and peritoneal microbiota by cervical mucus sampling in the general population.

Of note, the clear distinction between the upper and lower reproductive tract, intersecting at $\mathrm{CV}$ and $\mathrm{ET}$, remained intact even when performing an inter-individual comparison (Fig. 3d) (although the level of the inter-individual indices diminished from that of the intra-individual (Fig. 3c)), hence underscoring both anatomical as well as individual-specific features of the vagino-uterine microbiota.

Site-specific community types in the vagino-uterine microbiota. To further understand the relationship between communities at each site, we examined community types in all samples using the Dirichlet multinomial mixture (DMM) model ${ }^{5}$. Of the five community types detected by DMM (Supplementary Fig. 7), Type 1 and 5 were dominated by the Lactobacillus genus, which was present in notable amounts also in Type 2, 3 and 4 (Fig. 4a, b, Supplementary Data 7). Type 4, which contained a higher proportion of Prevotella and might correspond to the more diverse vaginal microbiota type reported previously ${ }^{3-5}$, was detected in some of the CV and ET samples but was not found in the FL and PF samples of this cohort (Fig. 4, Supplementary Fig. 7). The major type in PF, Type 2 was also present in CV, while Type 3 
a

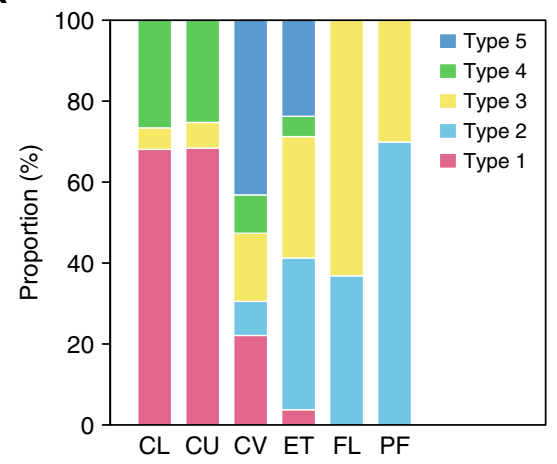

C

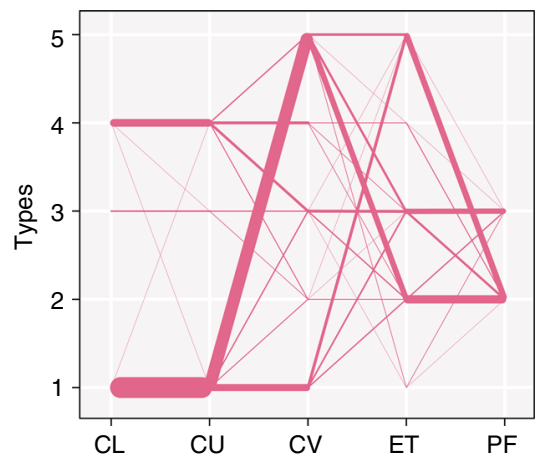

b

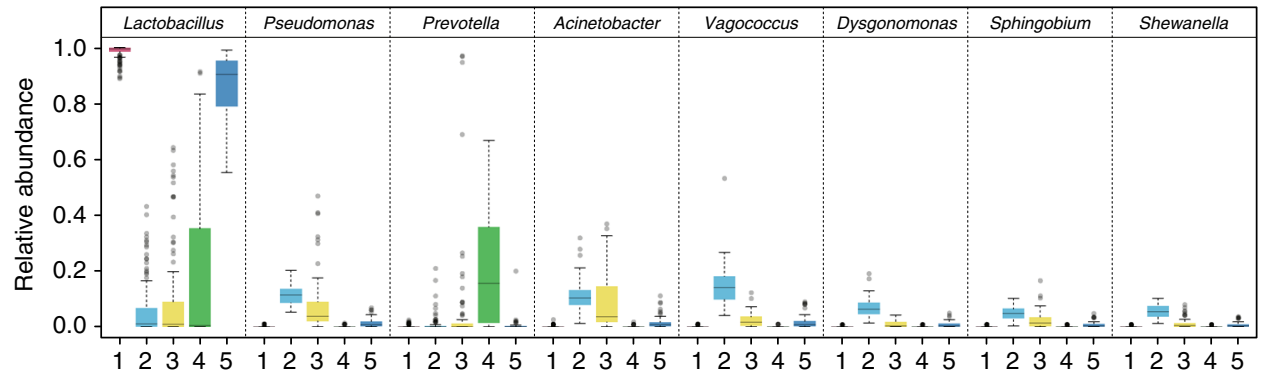

Fig. 4 Community types of the vagino-uterine microbiota. a Distribution of samples among the five community types at each body site $(n=94 \mathrm{CL}$, $95 \mathrm{CU}$, 95 CV, 80 ET, 93 PF, 9 FLL and 10 FRL, Supplementary Data 1). b Relative abundances of the top 8 most abundant genera in the 5 community types. The boxes denote the interquartile range (IQR) between the first and third quartiles (25th and 75th percentiles, respectively), and the line inside the boxes denote the median. The whiskers denote the lowest and highest values within 1.5 times the IQR from the first and third quartiles, respectively. Circles represent data points beyond the whiskers. c Community types connected between neighbouring sites according to individual subjects. Due to the small sample size, results for the fallopian tubes were plotted in a separate graph (Supplementary Fig. 7c). The width of each line indicates the number of subjects $(n=1-95)$

was present at all sites, yet dominating in the FL samples (Fig. 4, Supplementary Fig. 7).

When we examined transitions between community types at neighbouring sites, most subjects with Type 1 in the vagina (l CL) remained Type 1 at the $\mathrm{CU}$, and changed to Type 5 either at $\mathrm{CV}$ or at ET, and then turned to Type 2 in PF (Fig. 4c). By contrast, subjects with Type 3 or 4 at the vaginal sites rarely became Type 5 at the uterine sites, while some Type 4 subjects were found to switch to Type 3 in the upper part (Fig. 4b, Supplementary Fig. 7c). These findings corroborated our aforementioned results that there is an intra-individual continuum of the microbiota from the vagina to the peritoneal fluid that gradually changes according to the habitat.

Lifestyle and clinical factors associated with microbiota changes. The vaginal microbiota has previously been reported to vary between ethnic groups (in the US) and the menstrual cycle ${ }^{3,4}$. With our comprehensive collection of metadata (Supplementary Data 1), we examined whether various clinical and lifestyle factors were associated with changes in the vagino-uterine microbiota in women of reproductive age. Factors such as age of initial sexual intercourse, duration of menstrual period, and gravida and para appeared associated with microbiota composition at one or more sites, but failed to reach statistical significance after controlling for multiple testing (permutational multivariate analysis of variance (PERMANOVA), $P<0.05$ but $q>0.05$, Supplementary Data 8 and 9). Having given birth (multipara versus nullipara) was associated with microbiota composition in $\mathrm{CV}$ and $\mathrm{PF}$ $(q<0.05$, Supplementary Data 9$)$. Herbal medication was associated with microbiota composition in the CV only $(q<0.05$, Supplementary Data 9). Age, hysteromyoma, adenomyosis and endometrosis were also associated with microbiota composition in the PF $(q<0.05$, Supplementary Data 8,9$)$. Therefore, variations in the composition of the vagino-uterine microbiota are associated with a number of natural and diseased physiological conditions important for women's health.

Identification of diseases based on the vagino-uterine microbiota. We further examined whether variations in the vaginouterine microbiota composition were associated with different physiological conditions and diseases of the reproductive tract.

In relation to hysteromyoma (benign tumours in the uterus), Lactobacillus sp. were found to be more abundant in the vaginal and cervical samples of individuals with no hysteromyoma, while L. iners was more abundant in the $\mathrm{CV}$ of individuals with hysteromyoma (Supplementary Fig. 8). Among the hysteromyoma-enriched OTUs, one OTU in the CU and 11 OTUs in the PF were also identified to be influenced by the stage in the menstrual cycle (Spearman's correlation coefficient $>0.3$ or $<-0.3, q<0.05$, Supplementary Fig. 8); however, none of these OTUs showed a correlation with age. Overall, the results were consistent with hysteromyoma being associated with a minimally altered vagino-uterine microbiota.

Subjects with adenomyosis (abnormal presence of endometrial tissue within the myometrium) showed depletion or enrichment of many bacteria throughout the reproductive tract, some of which overlapped with bacteria associated with anaemia (Supplementary Figs. 9 and 10), which was consistent with clinical links between the two conditions $(P=0.001062$ between the two conditions in our cohort, Fisher's exact test). Furthermore, cross-validated random forest models distinguished subjects with adenomyosis from those without according to OTUs 
from any of the sites (Supplementary Fig. 11, Supplementary Data 10).

The microbiota-based models also allowed us to distinguish subjects without and with infertility attributed to endometriosis, a condition where endometrial cells present abnormal growth outside the uterus, and a major cause of infertility (Fig. 5, Supplementary Data 11). Altogether, these results suggest that the composition of the vagino-uterine microbiota potentially could be

a

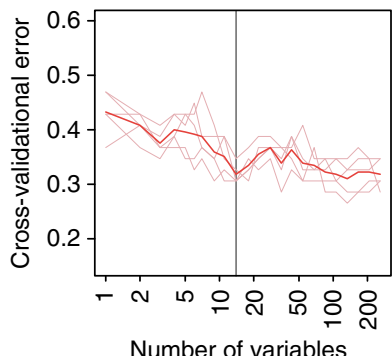

b

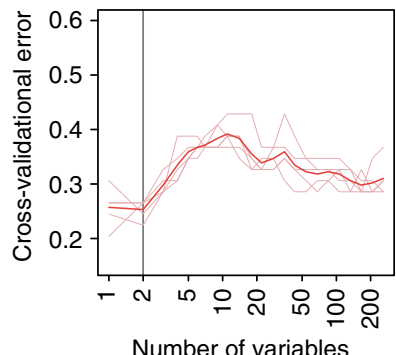

C

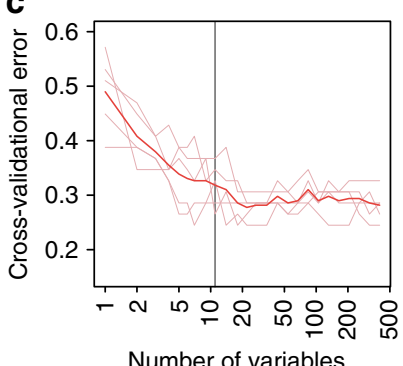

d

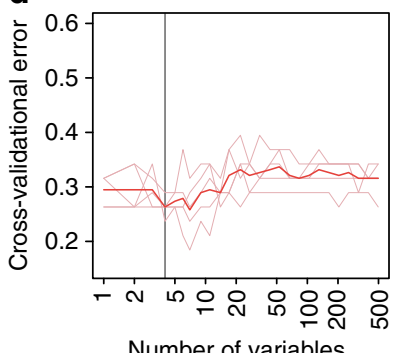

Number of variables

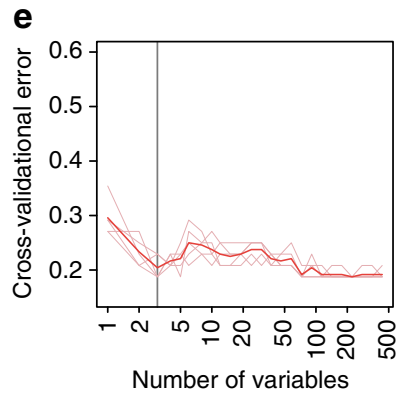

f

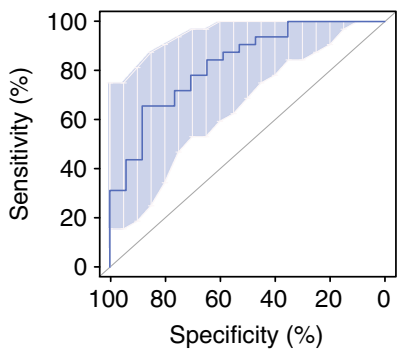

g

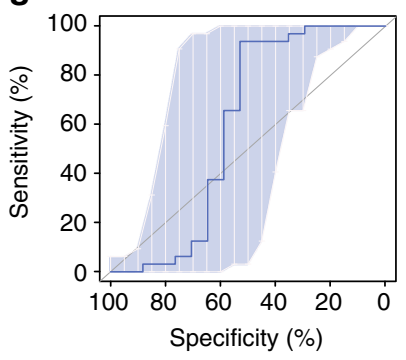

h

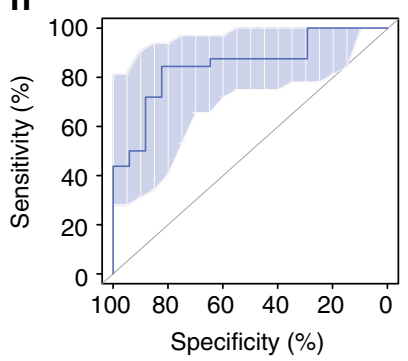

i
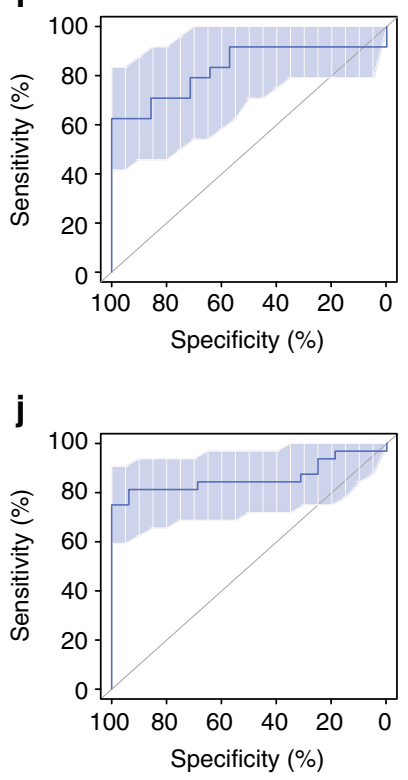

used to detect a number of ailments common to reproductive age women.

Functional inferences of the reproductive tract microbiota. To estimate the functional capacities of the vagino-uterine microbiota, we predicted KOs (Kyoto Encyclopedia of Genes and Genomes (KEGG) Orthology groups) from the 16S rRNA gene data.

In the case of adenomyosis, the analyses revealed that adenomyosis affected functions throughout the vagino-uterine microbiota (Supplementary Fig. 12). The microbiota in subjects with adenomyosis were especially enriched in pathways involved in flagella assembly and biosynthesis of aromatic amino acids, while the microbiota in subjects without adenomyosis were enriched in phosphotransferase system and fatty acid biosynthesis. These results suggest that functionally, the microbiota is also a continuum in the reproductive tract, and could be perturbed at multiple sites in a disease.

Menstrual cycle relates to the vagino-uterine microbiota and its function. Differences in the vagino-uterine microbiota were also identified between phases of the menstrual cycle, as was apparent from cross-validated random-forest models that classified samples into different phases of the cycle (Supplementary Fig. 13). OTUs that led to optimal classification included L. iners, and Lactobacillus sp. from vaginal sites, Sphingobium sp., Propionibacterium acnes and Pseudomonas sp. from the ET or PF, which were differentially enriched during the proliferative and secretory phases (Supplementary Fig. 13c). Notably, P. acnes was more abundant in the secretory phase in the ET (Supplementary Fig. 13l), and has previously been identified in the placenta and cultured from follicular fluid ${ }^{8,22-24}$. Functionally, the proliferative phase appeared associated with increased bacterial proliferation in the vagina and ET compared to the secretory phase, seen as higher abundance of pathways for pyrimidine and purine metabolism, aminoacyl-tRNA biosynthesis, and peptidoglycan biosynthesis (Fig. 6). The secretory phase, on the other hand, showed higher abundance of pathways for porphyrin metabolism, arginine and proline metabolism, degradation of benzoate, nitrotoluene and biosynthesis of siderophore (Fig. 6). These results indicate that the vagino-uterine microbiota probably varies according to the menstrual cycle.

\section{Discussion}

Herein we demonstrate the existence of distinct bacterial communities throughout the female reproductive tract forming a continuum of microbiotas changing from the vagina to the

Fig. 5 Microbiota-based classification of infertility due to endometriosis. $\mathbf{a}, \mathbf{b}, \mathbf{c}, \mathbf{d}, \mathbf{e}$ Distribution of 5 trials of 10 -fold cross-validation error in random forest classification of fertile versus infertile samples as the number of OTUs increases (a, CL; b, CU; c, CV; d, ET; e, PF). The model was trained using relative abundance of the OTUs (present in at least $10 \%$ of the samples) in the samples ( $n=16$ without endometriosis (fertile), 32 with endometriosis (infertile)). Subjects with endometriosis who had given birth, and infertile subjects due to reasons other than endometriosis, e.g. salpingemphraxis, were not analysed (Supplementary Data 1). The red curve indicates an average of the five trials (pink lines). The grey line marks the number of OTUs in the optimal set. $(\mathbf{f}, \mathbf{g}, \mathbf{h}, \mathbf{i}, \mathbf{j})$ Receiver operating curve (ROC) for the cross-validated sample set (f, CL; $\mathbf{g}, \mathrm{CU} ; \mathbf{h}, \mathrm{CV} ; \mathbf{i}, \mathrm{ET} ; \mathbf{j}$, $\mathrm{PF})$. The area under receiver operating curve $(A \cup C)$ is $0.8272,0.5919$, $0.8493,0.8304$ and 0.8613 , respectively. The $95 \%$ confidence intervals (CI) are shown as shaded areas. The diagonal lines mark an AUC of 0.5 (i.e. random classification). 


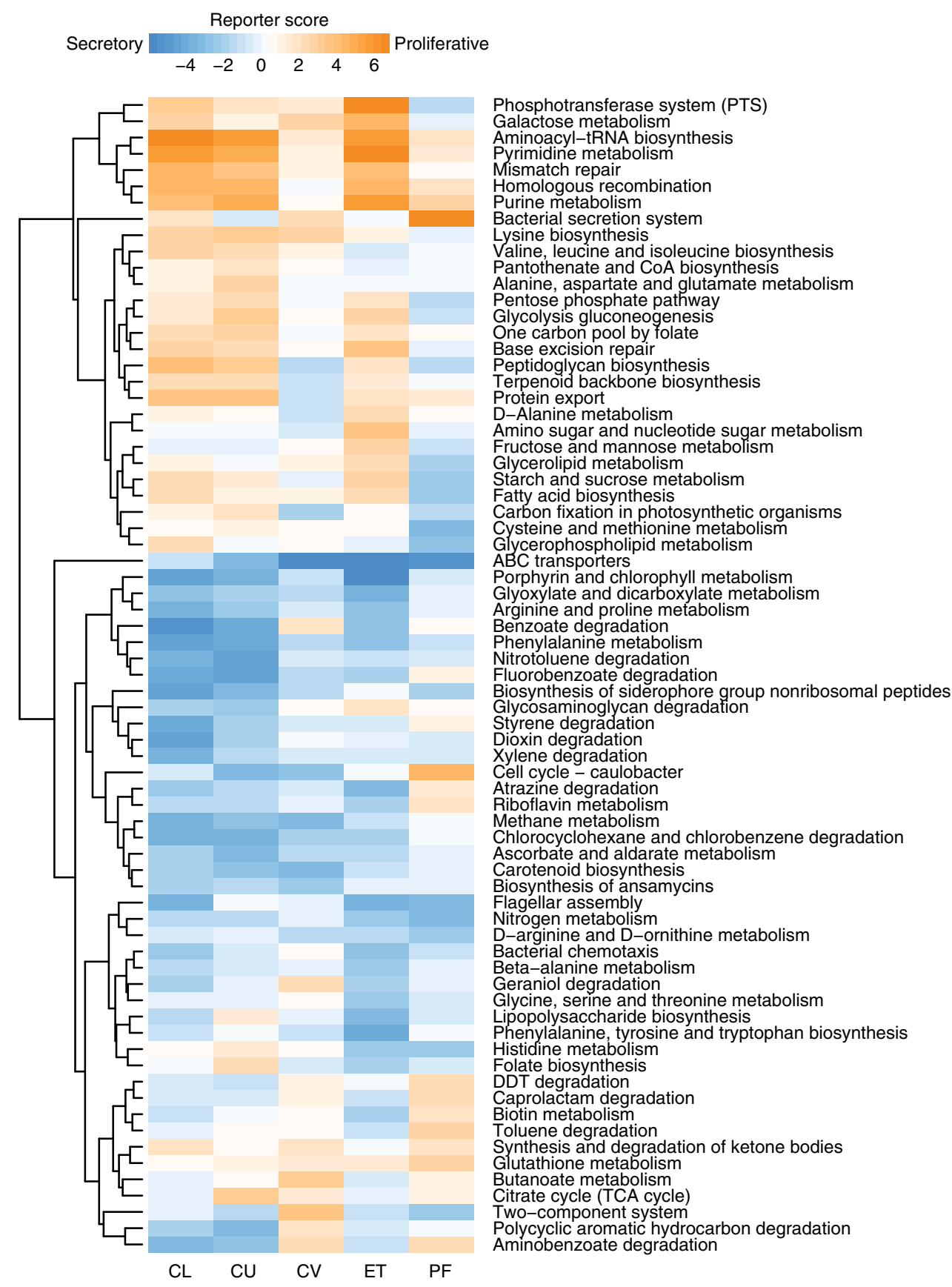

Fig. 6 Associations between inferred microbial functions and menstrual phases. Microbial KEGG pathways enriched in the proliferative or the secretory phases (orange versus blue, reporter score $>1.96$ or $<-1.96$ ) and present in all the body sites were plotted as a heatmap. The pathways were arranged by unsupervised hierarchical clustering. Samples derive from the study cohort of 95 reproductive-age women $(n=94 \mathrm{CL}, 95 \mathrm{CU}, 95 \mathrm{CV}, 80 \mathrm{ET}, 93 \mathrm{PF}$, 9 FLL and 10 FRL, Supplementary Data 1)

ovaries, which challenge the traditional view of human foetal development being a sterile event ${ }^{8,25-27}$. Indeed, vertical transmission of the mothers' microbiota before birth could be the norm throughout the animal kingdom, as previously alluded to by others ${ }^{28}$. Demonstrating a microbiota in traditionally unchartered territories remains a challenging task, and as illustrated here, the combination of high-throughput sequencing, quantitative PCR, culturing of live bacteria and other techniques would make a stronger case. Endosymbiotic, instead of free-living bacteria, is also an intriguing possibility.

As it is not possible to directly sample the upper reproductive tract (without going through the cervical os. and especially for FL, $\mathrm{PF}$ ) of fully healthy women, we have included a handful of fertile and infertile conditions that are not known to involve infection. The relevance of the vagino-uterine microbiota to female fecundity would require further analyses in large cohorts and in model systems. Although differing by disease cohorts and methodology, our sequencing-based analyses nevertheless showed some similarity to an early study on chronic endometritis ${ }^{29}$ (Supplementary Table 4) and a recent study on endometrial cancer $^{30}$ (Supplementary Table 5). Notably, the latter study also pointed to the exciting possibility of idendifying endometrial cancer through sampling the vaginal microbiota ${ }^{30}$. How disturbances to the vagino-uterine microbiota might eventually lead to benign or malignant conditions threatening the health of pre-menopausal and post-menopausal women is a major open question. 
Of relevance for clinical practice was the finding of intraindividual correlations between microbiota of the PF and that of $\mathrm{CV}$, as this indicates that (minimally invasive) sampling of cervical mucosa could be used to survey the status of the uterus and peritoneal cavity in the general population. This is of relevance in relation to the demonstrated associations between the uterine microbiota and diseases such as adenomyosis and endometrosis. We also showed that microbiota at the upper reproductive tract can be available through laparoscopy with minimal artificial effects and contaminations, laying the basis for the usage of this sampling route in subsequent studies.

We find that both the upper and the lower reproductive tract are home for facultative anaerobes and aerobes, in contrast to the situation in bacterial vaginosis (and more recently also reported in endometrial cancer ${ }^{30}$, Supplementary Table 5), represented by overgrowth with obligate anaerobes, being linked to adverse outcomes such as pre-term birth and sexually transmitted diseases $^{2}, 6,7$. Clothing, sedentary lifestyle, contraception and delayed pregnancies common in modern life may modulate the vagino-uterine microbiota. While vaginal Lactobacillus species are known to inhibit other bacteria by maintaining a high concentration of lactic acid and hydrogen peroxide production, a healthy uterine microbiota likely also depends on the nutrients and hormones available to the uterus, as well as the microbiota in the vagina and the peritoneal cavity ${ }^{1,31}$. In this regard, it was striking to identify associations between inferred microbial function and uterine-related diseases as well as to the menstrual cycle, which would require validations in larger and prospective cohorts $^{32}$

\section{Methods}

Study cohort and sample collection. The study was approved by the institutional review boards at Peking University Shenzhen Hospital and BGI-Shenzhen. As an initial cohort, 95 reproductive age women operated for conditions not known to involve infection (hysteromyoma, adenomyosis, endometriosis, salpingemphraxis) were included in the study by Peking University Shenzhen Hospital between December 2013 and July 2014 (Supplementary Data 1). Subjects with vaginal inflammation, severe pelvic adhesion, any acute inflammation, cancer, endocrine or autoimmune disorders were excluded. The subjects had no recorded recent use of hormones, antibiotics and vaginal medications, no cervical treatment within a week, no douching within 5 days and no sexual activity within $48 \mathrm{~h}$. None of the subjects were pregnant, lactating or in menses at the time of sampling. Informed consent were obtained from all participants.

Samples from the lower reproductive tract (CL, CU, CV) were taken on the day of the clinical visit with no prior perturbation. Samples from the upper reproductive tract (ET, FL, PF) were taken during operation (on average 2.81 days later, Supplementary Fig. 1, Supplementary Data 1). Laparoscopy was used except for subjects with adenomyosis who need laparotomy. Care was taken to avoid contamination by blood. PF was sampled at the Pouch of Douglas after sterile saline was injected into peritoneal cavity, and then the FL and ET were sampled upon opening.

Nylon flocked swabs (Chenyang Global Group, CY-93050 and CY-98000) were used for all samples except PF. The swab heads were then placed in sterile PBS and flash-frozen with liquid nitrogen, stored at $-80^{\circ} \mathrm{C}$ and transported on dry ice to BGI-Shenzhen.

DNA extraction and 16S rRNA amplicon sequencing. DNA extraction was performed as described ${ }^{33}$. Sample was treated with Lysozyme, proteinase $\mathrm{K}$ and SDS, then purified by phenol-chloroform-isoamylalcohol, precipitated by glycogen, sodium acetate and cold isopropanol, washed with $70 \%$ ethanol and resuspended in $1 \times$ TE buffer. The V4-V5 region of the $16 \mathrm{~S}$ rRNA genes was amplified by polymerase chain reaction (PCR) with a universal forward primer and a unique barcoded fusion reverse primer (V4-515F: 5'-GTGCCAGCMGCCGCGGTAA-3' and V5-907R: 5'- CCGTCAATTCMTTTRAGT-3', where $\mathrm{M}$ indicates A or C and $\mathrm{R}$ indicates purine).

PCR was performed using the following conditions: 3 min denaturation at $94^{\circ} \mathrm{C} ; 25$ cycles of denaturation at $94^{\circ} \mathrm{C}$ for $45 \mathrm{~s}$, annealing at $50{ }^{\circ} \mathrm{C}$ for $60 \mathrm{~s}$, elongation at $72{ }^{\circ} \mathrm{C}$ for $90 \mathrm{~s}$; and final extension at $72^{\circ} \mathrm{C}$ for $10 \mathrm{~min}$. The amplicons were purified using the AMPure beads (Axygen). Barcoded libraries were generated by emulsion PCR, and sequenced in a V5 to V4 reverse direction on a 318 chip using the $400 \mathrm{bp}$ sequencing kit of the Ion Torrent Personal Genome Machine (PGM) system according to manufacturer's instructions.
Processing of $16 \mathrm{~S}$ sequencing data. The raw data from the PGM system were exported and pre-processed by using Mothur (V1.33.3) ${ }^{34}$. The criteria for highquality reads included: (1) longer than $200 \mathrm{bp}$; (2) less than two mismatches with the degenerate PCR primers; (3) average quality score $<25$. OTUs based on the $16 \mathrm{~S}$ rRNA gene sequences were generated with the identity cutoff of $97 \%$ using QIIME's UCLUST program ${ }^{35}$. The seed sequences of each OTU were chosen for taxonomic classification against the Greengene reference sequences (gg_13_8_otus) by the UCLUST taxonomy assigner. The $\alpha$-diversities and $\beta$-diversities, Unifrac analyses were also calculated in QIIME with taxonomic abundance profiles at the OTU or genus level.

For OTU \#1 from our samples (an abundant OTU from the vagina), the seed sequence showed over $97 \%$ identity with the V4V5 region of both the Lactobacillus kitasatonis and Lactobacillus crispatus $16 \mathrm{~S}$ rRNA genes. To resolve this ambiguity, full-length 16S rRNA gene and a 289 bp fragment of the conserved single-copy gene encoding phenylalanine-tRNA ligase beta subunit (PF03147) were cloned from a sample containing 99\% OTU \#1 (C056CU), and Sanger sequencing (Applied Biosystems 3730 DNA analyzer) confirmed both genes to be from L. crispatus ST1 (NCBI gene ID: 9107847 and 9107287, respectively).

Identification of signature OTUs. Signature OTUs were identified according to their IndVal values, which consider both the occurrence and abundance of a $\operatorname{taxon}^{27,36}$

$$
\operatorname{IndVal}_{\mathrm{p}}=\frac{a_{\mathrm{p}}}{a} \times \frac{n_{\mathrm{p}}}{N_{\mathrm{p}}}
$$

where $a_{\mathrm{p}}$ is the sum of the abundances of species within the samples from body site $\mathrm{p}, n_{\mathrm{p}}$ is the number of occurrences of the species within the samples from site $\mathrm{p}, a$ is the sum of the abundances of species from all body sites, $N_{\mathrm{p}}$ is the number of samples from site $\mathrm{p}$.

Statistical tests on the IndVal values were performed as described previously, through permutation of the relative abundance of each genus or OTU across communities, using the multipatt function of the R package 'indicspeces ${ }^{27}$.

Concordance of the microbiota between samples. We used the Sorenson index (Sørensen-Dice index) to measure the similarity between microbiota at different body sites of the same individual, based on the presence and absence of OTUs. The index was calculated as:

$$
\mathrm{QS}=\frac{2 C}{A+B}=\frac{2|A \cap B|}{|A|+|B|}
$$

where $A$ and $B$ are the number of OTUs in two samples $A$ and $B$, respectively, and $C$ is the number of OTUs shared by the two samples; QS is the quotient of similarity, which ranges from 0 to 1 .

Community types of the vagino-uterine microbiota. Relative abundances of genera in all 476 samples were used to determine the optimal number of clusters according to $\mathrm{DMM}^{5}$. Transitions between community types were assessed statistically from community type assignments for all subjects together, as well as for those who had fallopian tube samples.

PERMANOVA on the influence of phenotypes. We applied PERMANOVA on the relative abundances of OTUs or metabolites in the samples to assess impact from each of the factors listed 37,38 . We used Bray-Curtis or UniFrac distance and 9999 permutations in $\mathrm{R}\left(3.0 .0\right.$, vegan package $\left.{ }^{38,39}\right)$.

Random-forest classification. In order to construct a model that could distinguish samples of different menstrual phases for each body site, the relative abundances of OTUs (found in at least $10 \%$ of the samples) in the samples were fitted against the samples' actual days in the menstrual cycle using default parameters in the randomForest package in $\mathrm{R}(3.1 .2 \mathrm{RC})$ except that 1000 trees were used, as in previous reports on the gut microbiota age of infants ${ }^{27}, 40$. Ten-fold cross-validation was performed five times. The cross-validation error curves from the five trials were averaged, and the minimum error in the averaged curve plus the standard deviation at that point were used as the cutoff for acceptable error. From the sets of OTUs with a classification error less than the cutoff, the set with the smallest number of OTUs was chosen as the optimal set ${ }^{38,41}$.

Random-forest classification of samples according to non-continuous phenotypes was performed in the same manner.

MetaScope in the Progenesis QI software was used to search for compound identifications not only based on neutral mass, isotope distribution and retention time, but also based on collisional cross-sectional area and MS/MS fragmentation data in the HMDB database.

Functional inference of $\mathbf{1 6 S}$ data. PICRUSt (phylogenetic investigation of communities by reconstruction of unobserved states) was used to infer KOs from OTU data as previously described ${ }^{42}$. The relative abundance of KEGG pathways 
and modules was summed from the relative abundance of KOs belonging to these pathways and modules.

Differentially enriched KEGG pathways or modules were identified according to their reporter score ${ }^{27,38,43}$, from the $Z$-scores of individual KEGG orthology groups. A reporter score of $Z=1.96$ was used as a detection threshold for pathways or modules that were significantly over-represented in one group relative to the other, corresponding to $95 \%$ confidence.

Real-time quantitative PCR. Four major vaginal Lactobacillus species including $L$. crispatus, L.iners, $L$ jensenii and $L$. gasseri were quantified by real-time $\mathrm{qPCR}$ modified from a previously described regimen ${ }^{44}$. After DNA quantification was conducted for each sample using Qubit Fluorometer (Life Technologies), $1.6 \mu \mathrm{l}$ DNA solution was used as the template for real-time PCR. Standard curves were generated using serial tenfold dilutions of plasmids. The range of amplification efficiency for the qPCR was from 90 to $110 \%$, and linearity values were all $\geq 0.99$. Real-time qPCR was conducted using SYBR Premix Ex Taq GC (TAKARA) using the StepOnePlus Real-time PCR System (Life Technologies). Each reaction included a standard curve, and each sample was run in triplicate and contained $10 \mu \mathrm{l}$ SYBR Premix Ex Taq GC, $1.6 \mu \mathrm{l}$ of DNA template along with $0.2 \mu \mathrm{M}$ primers in a final reaction volume of $20 \mu \mathrm{l}$. Sterile PBS and sterile physiological saline were used as diluent negative controls, which were subject to sample processing, DNA extraction and real-time qPCR in parallel with samples according to our standard protocol, in order to determine the source of contamination. In addition, nontemplate control, which was ultrapure water, was also included when performing real-time qPCR. Total DNA amount for each sample was calculated by multiplying the concentration of DNA by the total volume of extracted DNA.

\section{Validation cohort used for control of contamination and bacterial culturing} We used an independent validation cohort comprised of additional 15 reproductive age women included between May 2016 and June 2016 (Supplementary Data 1). The hospital and conditions for surgery were the same as those in study cohort. Informed consent were also obtained from all participants.

Samples from the upper reproductive tract (ET, FL, PF) were collected during operation. For each operation, sterile PBS, sterile physiological saline, dry sterile swabs rubbed on patient's preoperative skin area, dry sterile swabs rubbed on surgeon's gloved fingers were sampled as diluent negative controls. The swab heads were then placed in sterile PBS (Supplementary Fig. 1).

PF samples and diluent negative controls from the additional 15 women were collected for cultivation experiment. All the samples were kept under anaerobic conditions, and processed in the laboratory within $6 \mathrm{~h}$ of sample collection to maximise recovery of bacterial viability. In total, $100 \mu \mathrm{l}$ of peritoneal fluid specimens and $100 \mu \mathrm{l}$ of each diluent negative controls were plated on PYG agar (DSMZ 104 medium) with 5\% horse blood and incubated aerobically or anaerobically at $37^{\circ} \mathrm{C}$ for up to $72 \mathrm{~h}$. Reducing agents such as resazurin and cysteine- $\mathrm{HCl}$ were not added to cultures incubated anaerobically. Bacterial isolates from the plates were selected based on morphologic characteristics. DNA from single colonies was extracted using the Bacterial DNA Kit (OMEGA) and then amplified using the universal primers $27 \mathrm{~F} / 1492 \mathrm{R}^{45}$. The amplicons were purified using the Magbind PCR Purification Kit (GeneOn BioTeach) and then sent for Sanger sequencing. The sequences were analysed using the GenBank 16S rRNA sequences database using the BLAST algorithm, and sequences with more than $97 \%$ similarity were considered to be of the same species.

Data availability. Sequence reads for the 544 samples have been deposited in the European Nucleotide Archive under study numbers PRJEB16013 and PRJEB21098. The authors declare that all other relevant data supporting the findings of the study are available in this article and its Supplementary Information files, or from the corresponding author upon request.

Received: 17 March 2017 Accepted: 3 August 2017

Published online: 17 October 2017

\section{References}

1. Brunelli, R. et al. Globular structure of human ovulatory cervical mucus. FASEB J. 21, 3872-3876 (2007).

2. Ma, B., Forney, L. J. \& Ravel, J. Vaginal microbiome: rethinking health and disease. Annu. Rev. Microbiol. 66, 371-389 (2012).

3. Ravel, J. et al. Vaginal microbiome of reproductive-age women. Proc. Natl Acad. Sci. USA 108, 4680-4687 (2010).

4. Gajer, P. et al. Temporal dynamics of the human vaginal microbiota. Sci. Transl. Med. 4, 132ra52-132ra52 (2012).

5. Ding, T. \& Schloss, P. D. Dynamics and associations of microbial community types across the human body. Nature 509, 357-360 (2014).

6. Goldenberg, R. L., Hauth, J. C. \& Andrews, W. W. Intrauterine infection and preterm delivery. N. Engl. J. Med. 342, 1500-1507 (2000).

7. Hyman, R. W. et al. Diversity of the vaginal microbiome correlates with preterm birth. Reprod. Sci. doi:10.1177/1933719113488838 (2013).
8. Aagaard, K. et al. The placenta harbors a unique microbiome. Sci. Transl. Med 6, 237ra65 (2014).

9. Salter, S. J. et al. Reagent and laboratory contamination can critically impact sequence-based microbiome analyses. BMC Biol. 12, 87 (2014).

10. Vásquez, A., Jakobsson, T., Ahrné, S., Forsum, U. \& Molin, G. Vaginal Lactobacillus Flora of healthy Swedish women. J. Clin. Microbiol. 40, 2746-2749 (2002).

11. Gibbs, R. S., Blanco, J. D., St Clair, P. J. \& Castaneda, Y. S. Quantitative bacteriology of amniotic fluid from women with clinical intraamniotic infection at term. J. Infect. Dis. 145, 1-8 (1982).

12. Jiménez, E. et al. Isolation of commensal bacteria from umbilical cord blood of healthy neonates born by cesarean section. Curr. Microbiol. 51, 270-274 (2005).

13. Hogue, R., Graves, M., Moler, S. \& Janda, J. M. Pink-pigmented non-fermentative gram-negative rods associated with human infections : a clinical and diagnostic challenge. Infection 35, 126-133 (2007).

14. Flynn, A. N., Lyndon, C. A. \& Church, D. L. Identification by 16 S rRNA gene sequencing of an Actinomyces hongkongensis isolate recovered from a patient with pelvic actinomycosis. J. Clin. Microbiol. 51, 2721-2723 (2013).

15. Chang, D.-H., Rhee, M.-S. \& Kim, B.-C. Dermabacter vaginalis sp. nov., isolated from human vaginal fluid. Int. J. Syst. Evol. Microbiol. 66, 1881-1886 (2016).

16. McCormack, W. M. et al. Vaginal colonization with Corynebacterium vaginale (Haemophilus vaginalis). J. Infect. Dis. 136, 740-745 (1977).

17. Holcombe, L. J. et al. Pseudomonas aeruginosa secreted factors impair biofilm development in Candida albicans. Microbiology 156, 1476-1485 (2010).

18. Ligon, J. M. et al. Natural products with antifungal activity from Pseudomonas biocontrol bacteria. Pest Manag. Sci. 56, 688-695 (2000).

19. Kim, T. K. et al. Heterogeneity of vaginal microbial communities within individuals. J Clin Microbiol. 47, 1181-1189 (2009).

20. Yu, R. R. et al. A Chinese rhesus macaque (Macaca mulatta) model for vaginal Lactobacillus colonization and live microbicide development. J. Med. Primatol. 2, 125-136 (2009).

21. Albert, A. Y. K. et al. A study of the vaginal microbiome in healthy Canadian women utilizing cpn60-based molecular profiling reveals distinct Gardnerella subgroup community state types. PLoS ONE 10, 1-21 (2015).

22. Pelzer, E. S. et al. Hormone-dependent bacterial growth, persistence and biofilm formation - a pilot study investigating human follicular fluid collected during IVF cycles. PLoS ONE 7, e49965 (2012).

23. Pelzer, E. S. et al. TUNEL analysis of DNA fragmentation in mouse unfertilized oocytes: The effect of microorganisms within human follicular fluid collected during IVF cycles. J. Reprod. Immunol. 99, 69-79 (2013).

24. Pelzer, E. S. et al. Microorganisms within human follicular fluid: effects on IVF. PLoS ONE 8, e59062 (2013).

25. Mackie, R. I., Sghir, A. \& Gaskins, H. R. Developmental microbial ecology of the neonatal gastrointestinal tract. Am. J. Clin. Nutr. 69, 1035S-1045S (1999).

26. Matamoros, S., Gras-Leguen, C., Le Vacon, F., Potel, G. \& De La Cochetiere, M. F. Development of intestinal microbiota in infants and its impact on health. Trends Microbiol. 21, 167-173 (2013).

27. Bäckhed, F. et al. Dynamics and stabilization of the human gut microbiome during the first year of life. Cell Host Microbe 17, 690-703 (2015).

28. Funkhouser, L. J. \& Bordenstein, S. R. Mom knows best: the Universality of maternal microbial transmission. PLoS Biol. 11, e1001631 (2013).

29. Cicinelli, E. et al. Chronic endometritis: correlation among hysteroscopic, histologic, and bacteriologic findings in a prospective trial with 2190 consecutive office hysteroscopies. Fertil. Steril. 89, 677-684 (2008).

30. Walther-António, M. R. S. et al. Potential contribution of the uterine microbiome in the development of endometrial cancer. Genome Med 8, 122 (2016).

31. Huang, B., Fettweis, J. M., Brooks, J. P., Jefferson, K. K. \& Buck, Ga The changing landscape of the vaginal microbiome. Clin. Lab. Med. 34, 747-761 (2014).

32. Wang, J. \& Jia, H. Metagenome-wide association studies: fine-mining the microbiome. Nat. Rev. Microbiol. 14, 508-522 (2016).

33. Qin, J. et al. A metagenome-wide association study of gut microbiota in type 2 diabetes. Nature 490, 55-60 (2012).

34. Schloss, P. D. et al. Introducing mothur: open-source, platform-independent, community-supported software for describing and comparing microbial communities. Appl. Environ. Microbiol. 75, 7537-7541 (2009).

35. Caporaso, J. G. et al. QIIME allows analysis of high-throughput community sequencing data. Nat. Methods 7, 335-336 (2010).

36. Dufrêne, M. \& Legendre, P. Species assemblages and indicator species:the need for a flexible asymmetrical approach. Ecol. Monogr. 67, 345-366 (1997).

37. Anderson, M. J. A new method for non-parametric multivariate analysis of variance. Aust. Ecol. 26, 32-46 (2001).

38. Feng, Q. et al. Gut microbiome development along the colorectal adenoma-carcinoma sequence. Nat. Commun. 6, 6528 (2015). 
39. Zapala, M. A. \& Schork, N. J. Multivariate regression analysis of distance matrices for testing associations between gene expression patterns and related variables. Proc. Natl Acad. Sci. USA 103, 19430-19435 (2006).

40. Subramanian, S. et al. Persistent gut microbiota immaturity in malnourished Bangladeshi children. Nature 509, 417-421 (2014).

41. Zhang, X. et al. The oral and gut microbiomes are perturbed in rheumatoid arthritis and partly normalized after treatment. Nat. Med. 21, 895-905 (2015).

42. Langille, M. G. I. et al. Predictive functional profiling of microbial communities using 16S rRNA marker gene sequences. Nat. Biotechnol. 31, 814-821 (2013).

43. Patil, K. R. \& Nielsen, J. Uncovering transcriptional regulation of metabolism by using metabolic network topology. Proc. Natl Acad. Sci. USA 102, 2685-2689 (2005)

44. De Backer, E. et al. Quantitative determination by real-time PCR of four vaginal Lactobacillus species, Gardnerella vaginalis and Atopobium vaginae indicates an inverse relationship between L. gasseri and L. iners. BMC Microbiol. 7, 115 (2007).

45. Augustinos, A. A. et al. Exploitation of the medfly gut microbiota for the enhancement of sterile insect technique: Use of Enterobacter sp. in larval diet-based probiotic applications. PLOS ONE 10, 1-17 (2015).

\section{Acknowledgements}

The study was supported by the Shenzhen Municipal Government of China (JCYJ20160229172757249, JCYJ20150601090833370), the Danish Strategic Research Council grant (2106-07-0021), the Ole Rømer grant from Danish Natural Science Research Council and Solexa project (272-07-0196). We gratefully acknowledge colleagues at BGI-Shenzhen for DNA quality control, library construction, sequencing and helpful discussions.

\section{Author contributions}

H.Z., J.L., H.J. and R.W. conceived and directed the project. W.W., J.D., L.Z., H.D., H.T. and R.W. performed the clinical diagnosis, sample collection and result analyses., C.C., X.S., Z.L., F.L., Z.W., H.Xie, C.X., Y.X. and H.Xia performed the bioinformatic analyses, molecular biology experiments and prepared display items. X.C., C.Z. and B.W. analysed the metabolome. C.C., X.S., H.Z., X.Y., H.J., K.K. and S.B. wrote the manuscript. All authors contributed to the revision of the manuscript.

\section{Additional information}

Supplementary Information accompanies this paper at doi:10.1038/s41467-017-00901-0.

Competing interests: The authors declare no competing financial interests.

Reprints and permission information is available online at http://npg.nature.com/ reprintsandpermissions/

Publisher's note: Springer Nature remains neutral with regard to jurisdictional claims in published maps and institutional affiliations.

\begin{abstract}
(cc) (1) Open Access This article is licensed under a Creative Commons Attribution 4.0 International License, which permits use, sharing, adaptation, distribution and reproduction in any medium or format, as long as you give appropriate credit to the original author(s) and the source, provide a link to the Creative Commons license, and indicate if changes were made. The images or other third party material in this article are included in the article's Creative Commons license, unless indicated otherwise in a credit line to the material. If material is not included in the article's Creative Commons license and your intended use is not permitted by statutory regulation or exceeds the permitted use, you will need to obtain permission directly from the copyright holder. To view a copy of this license, visit http://creativecommons.org/ licenses/by/4.0/.
\end{abstract}

(C) The Author(s) 2017 